\title{
Review Article \\ Pharmacologic Prophylaxis of Portal Venous System Thrombosis after Splenectomy: A Meta-Analysis
}

\author{
Xingshun Qi, ${ }^{1,2}$ Ming Bai, ${ }^{2}$ Xiaozhong Guo, ${ }^{1}$ and Daiming Fan ${ }^{2}$ \\ ${ }^{1}$ Department of Gastroenterology, General Hospital of Shenyang Military Area, Shenyang 110840, China \\ ${ }^{2}$ Xijing Hospital of Digestive Diseases, Fourth Military Medical University, Xian, Shaanxi 710032, China \\ Correspondence should be addressed to Xiaozhong Guo; guo_xiao_zhong@126.com and Daiming Fan; fandaim@fmmu.edu.cn
}

Received 13 June 2014; Accepted 18 July 2014; Published 27 August 2014

Academic Editor: Valerio De Stefano

Copyright (C) 2014 Xingshun Qi et al. This is an open access article distributed under the Creative Commons Attribution License, which permits unrestricted use, distribution, and reproduction in any medium, provided the original work is properly cited.

\begin{abstract}
Portal venous system thrombosis (PVST) is a life-threatening complication of splenectomy. A meta-analysis was conducted to explore the role of pharmacologic prophylaxis of PVST after splenectomy. Overall, 359 papers were initially identified via the PubMed, EMBASE, and Cochrane Library databases. Eight of them were eligible. The incidence of PVST after splenectomy was significantly lower in patients who received the preventive measures than in those who did not (odds ratio [OR]: 0.33, 95\% confidence interval [CI]: $0.22-0.47, P<0.00001)$. Subgroup analyses demonstrated that the significant difference remained in studies including patients with portal hypertension $(n=6)$, but not in those including patients with hematological diseases $(n=2)$; the significant difference remained in studies using any type of prophylactic drugs (anticoagulants $[n=6]$, thrombolytics [ $n=1]$, and prostaglandin E1 $[n=1])$; the significant difference remained in nonrandomized studies $(n=5)$, but not in randomized studies $(n=3)$. The risk of bleeding was similar between the two groups (OR: $0.65,95 \%$ CI: $0.10-4.04, P=0.64)$. In conclusion, pharmacologic prophylaxis might decrease the incidence of PVST after splenectomy in patients with portal hypertension and did not increase the risk of bleeding. However, the effect of pharmacologic prophylaxis of PVST in patients with hematological diseases remained questioned.
\end{abstract}

\section{Introduction}

Portal venous system thrombosis (PVST) is a life-threatening vascular disease characterized by the development of thrombosis within the portal vein, mesenteric vein, and splenic vein $[1,2]$. Splenectomy is one of the most common local risk factors of PVST $[1,2]$. The incidence of PVST after splenectomy varies from $0 \%$ to $50 \%$ [3-8]. The heterogeneity is primarily attributable to the different sample sizes, surgical approaches, indications for splenectomy, and diagnostic methods of PVST among studies. The possible mechanism of PVST after splenectomy is the local injury to the vein and its secondary coagulation abnormalities [2]. In addition, the presence of hypercoagulability, weight of spleen, and diameter of splenic vein are considered as the major predisposing factors of PVST after splenectomy.

Pharmacologic prophylaxis may be helpful to decrease the incidence of PVST after splenectomy, thereby reducing its related morbidity and mortality. However, the risk of bleeding secondary to the use of anticoagulants or thrombolytics immediately after splenectomy should be cautioned [9-11], especially in cirrhotic patients with portal hypertension and hypersplenism. Until now, no study has systematically evaluated the efficacy and safety of the pharmacologically preventive measures for the development of PVST after splenectomy. Herein, we conducted a systematic review and meta-analysis to explore this issue.

\section{Methods}

This work was performed according to the PRISMA statement for reporting systematic reviews and meta-analyses of studies that evaluate health care interventions [12].

2.1. Search Strategy and Selection Criteria. We performed the literature search via the PubMed, EMBASE, and Cochrane Library databases (from the database inception to October 30, 2013). Additional relevant literature was identified by 
hand-searching the reference lists of identified literature. Search items were listed as follows: ("anticoagulation" [All Fields] or "anticoagulant" [All Fields] or "warfarin" [All Fields] or "heparin" [All Fields] or "low molecular weight heparin" [All Fields] or "LMWH" or "enoxaparin" [All Fields] or "thrombolysis" [All Fields] or "thrombolytic" [All Fields] or "lytic" [All Fields] or "urokinase" [All Fields] or "streptokinase" [All Fields] or "antithrombotic" [All Fields]) AND ("splenectomy" [All Fields]) AND ("splenic vein" [All Fields] or "splenic venous" [All Fields] or "portal vein" [All Fields] or "portal venous" [All Fields] or "mesenteric vein" [All Fields] or "mesenteric venous" [All Fields]) AND ("thrombosis" [All Fields] or "thrombus" [All Fields] or "thrombotic" [All Fields] or "occluded" [All Fields] or "occlusive" [All Fields] or "occlusion" [All Fields] or "obstructed" [All Fields] or "obstructive" [All Fields] or "obstruction" [All Fields]).

We selected the papers according to the following eligibility criteria. (1) Both randomized controlled trials and nonrandomized studies were considered, if the incidence of PVST after splenectomy was compared between patients who received the preventive measures and those who did not. (2) Narrative reviews, systematic reviews, meta-analyses, comments, editorials, animal studies, and case reports were excluded. (3) Studies unrelated to the prevention of PVST after splenectomy were excluded. (4) Studies without any detailed information regarding the prevention of PVST after splenectomy were excluded. (5) Studies with all included patients receiving the prevention of PVST after splenectomy were excluded. (6) There was no publication date, publication language, or publication status restriction.

2.2. Data Extraction. We extracted the following data into Excel tables, including the author, journal, publication year, region where a study was conducted, period of enrollment, study design, study population, type of surgery, information regarding the prevention of PVST after splenectomy, type and number of participants, inclusion and exclusion criteria, demographic data (age and sex), type of underlying diseases, liver function (the data was collected in patients with liver diseases, if available), incidence, location, degree of PVST after splenectomy, and incidence of bleeding after the implementation of pharmacologic prophylaxis. We also contacted the authors about the data that were not shown in their papers.

2.3. Assessment of Study Quality. We used the Cochrane Collaboration's tool version 5.1.0 and Newcastle-Ottawa scale to evaluate the quality of randomized and nonrandomized studies, respectively.

The Cochrane Collaboration's tool for assessing the risk of bias includes 6 domains, such as selection bias (i.e., random sequence generation and allocation concealment), performance bias (i.e., blinding of participants and personnel), detection bias (i.e., blinding of outcome assessment), attrition bias (i.e., incomplete outcome data), reporting bias (i.e., selective reporting), and other biases (i.e., other sources of bias). For each entry, a study can be judged as low, high, or unclear risk of bias.
The Newcastle-Ottawa scale includes 3 categories, such as selection, comparability, and outcome [13]. For each item within the selection and exposure categories, a study can be awarded a maximum of one star; for the comparability category, a study can be awarded a maximum of two stars.

Study quality was independently assessed by two authors. When there were any disagreements, a consensus was reached by discussion with each other.

2.4. Statistical Analysis. The number of PVST and bleeding events and total observed participants in two groups were extracted from each study. Then, odds ratio (OR) with 95\% confidence interval (CI) was calculated. Finally, the OR of each study was pooled, using either fixed-effects (Mantel-Haenszel method) [14] or random-effects model (DerSimonian-Laird method) [15]. When the heterogeneity among studies was not significant, we used the fixed-effects model to calculate the pooled data. Otherwise, we used the random-effects model. Additionally, subgroup analyses were performed to identify the efficacy of pharmacologic prophylaxis according to the type of study population (hematological diseases or portal hypertension), type of drugs used for the prevention of PVST (anticoagulants, thrombolytics, or others), and study design (randomized or nonrandomized studies). Heterogeneity among studies was assessed by using the $I^{2}$ statistic $\left(I^{2}>50 \%\right.$ was considered as having substantial heterogeneity) and the Chi-square test $(P<0.10$ was considered to represent significant statistical heterogeneity) [16]. Sensitivity analyses were performed by sequential omission of every individual study to explore the cause of heterogeneity among studies. Funnel plots were used to assess the publication bias. All analyses were conducted using the statistical package Review Manager version 5.1 (Copenhagen, The Nordic Cochrane Center, The Cochrane Collaboration, 2011).

\section{Results}

Overall, 359 papers were initially identified. After exclusion, 8 papers were included in our meta-analysis (Figure 1) [1724]. Notably, a randomized controlled trial by Wang et al. was terminated due to the absence of funding support [23]. According to the preplanned study protocol, the study would require 214 participants (107 per arm). However, only 35 patients were finally enrolled. Additionally, in a retrospective study by Lai et al., regular and irregular anticoagulation were considered as the exposed and nonexposed group, respectively [19]. In detail, regular anticoagulation referred to subcutaneous injection of LMWH followed by oral warfarin; by comparison, irregular anticoagulation referred to aspirin or warfarin monotherapy for an undesignated time period without LMWH. Given the significant difference of drugs used between the two groups, this study was considered eligible to evaluate the effect of anticoagulation for the prevention of PVST after splenectomy.

These included studies were published in full-texts $(n=$ 7) or abstract $(n=1)$ between 2000 and 2013 (Table 1). Of them, 3 were randomized trials [20, 23, 24], 2 were prospective cohort studies $[17,18]$, and 3 were retrospective 


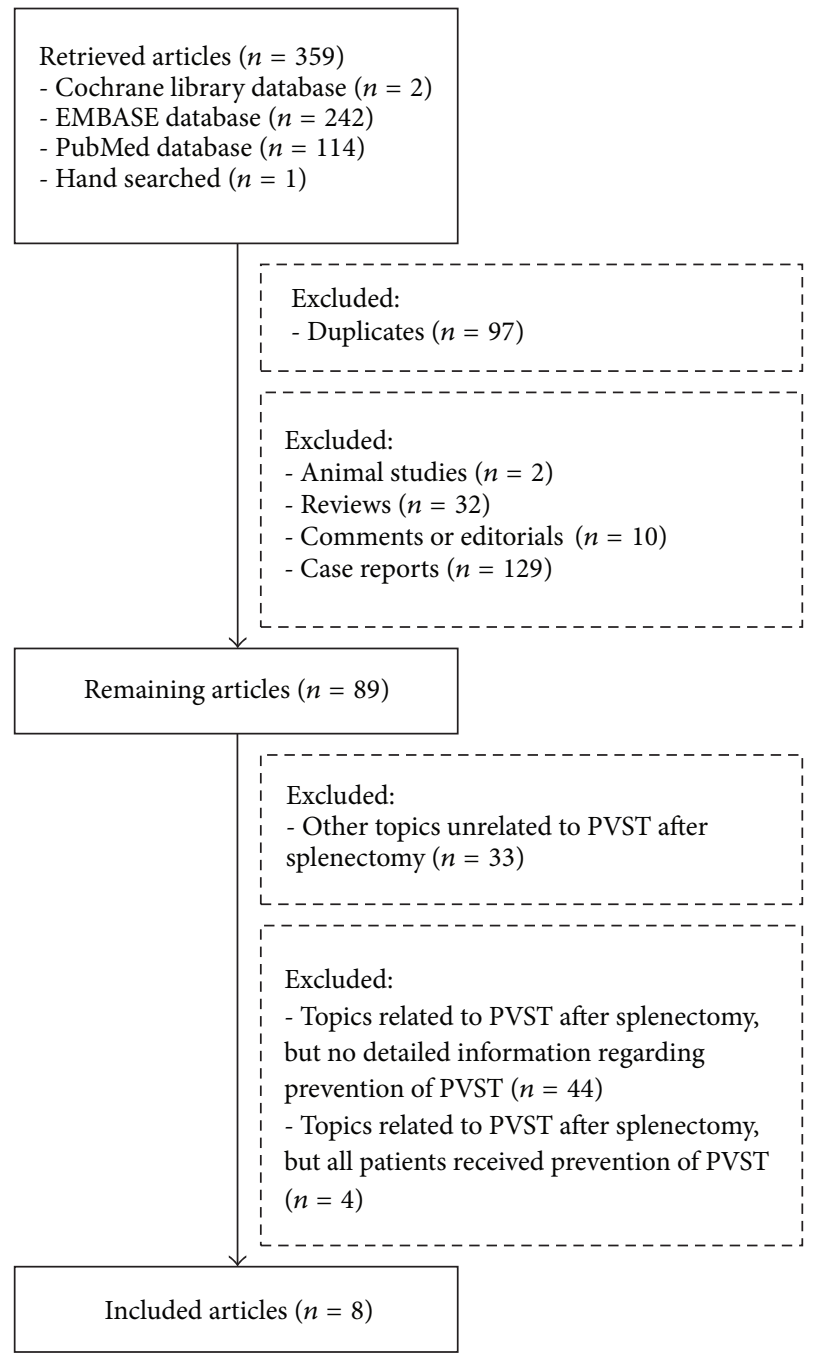

FIGURE 1: Flowchart of study inclusion.

cohort studies $[19,21,22]$. These studies were conducted by the investigators from Canada $(n=1)$, China $(n=4)$, Japan $(n=2)$, and Sweden $(n=1)$. In 6 Asian studies from China and Japan, the patients undergoing splenectomy were diagnosed with liver cirrhosis, portal hypertension, and/or hypersplenism [17-21, 24]. In 2 Western studies from Canada and Sweden, the patients undergoing splenectomy were diagnosed with hematological diseases [22, 23]. The information regarding the eligibility criteria of patients was summarized in Supplementary Table 1 available online at http://dx.doi.org/10.1155/2014/292689. The detailed information regarding the prevention of PVST after splenectomy was summarized in Supplementary Table 2. Among them, anticoagulants were employed in 6 studies, thrombolytics in 1 study, and prostaglandin E1 in 1 study.

Patient characteristics of these included studies were summarized in Supplementary Table 3. Although we contacted Ma and Pan for the data regarding sex, age, and liver function, none replied. Among these studies, 2 studies did not provide the data regarding the sex and age [20,21], and 1 study including patients with portal hypertension did not provide the data regarding liver function [21].

\subsection{Study Quality}

3.1.1. Randomized Studies. For the selection bias, 1 and 2 studies were at a low and unclear risk, respectively (Supplementary Table 4). For the performance, 2 studies were at an unclear risk, and another study was at a high risk because it was an open-label study. For the detection bias, 3 studies were at a low risk. For the attrition bias, 2 studies were at an unclear risk, and another study was at a high risk because it was an underpowered study. For the reporting and other biases, all of the 3 studies had an unclear risk.

3.1.2. Nonrandomized Studies. Two and 3 studies were awarded $<5$ and $\geqq 5$ stars, respectively (Supplementary Table 5).

3.2. Incidence of PVST. All of the 8 included studies provided the data regarding the incidence of PVST after splenectomy 


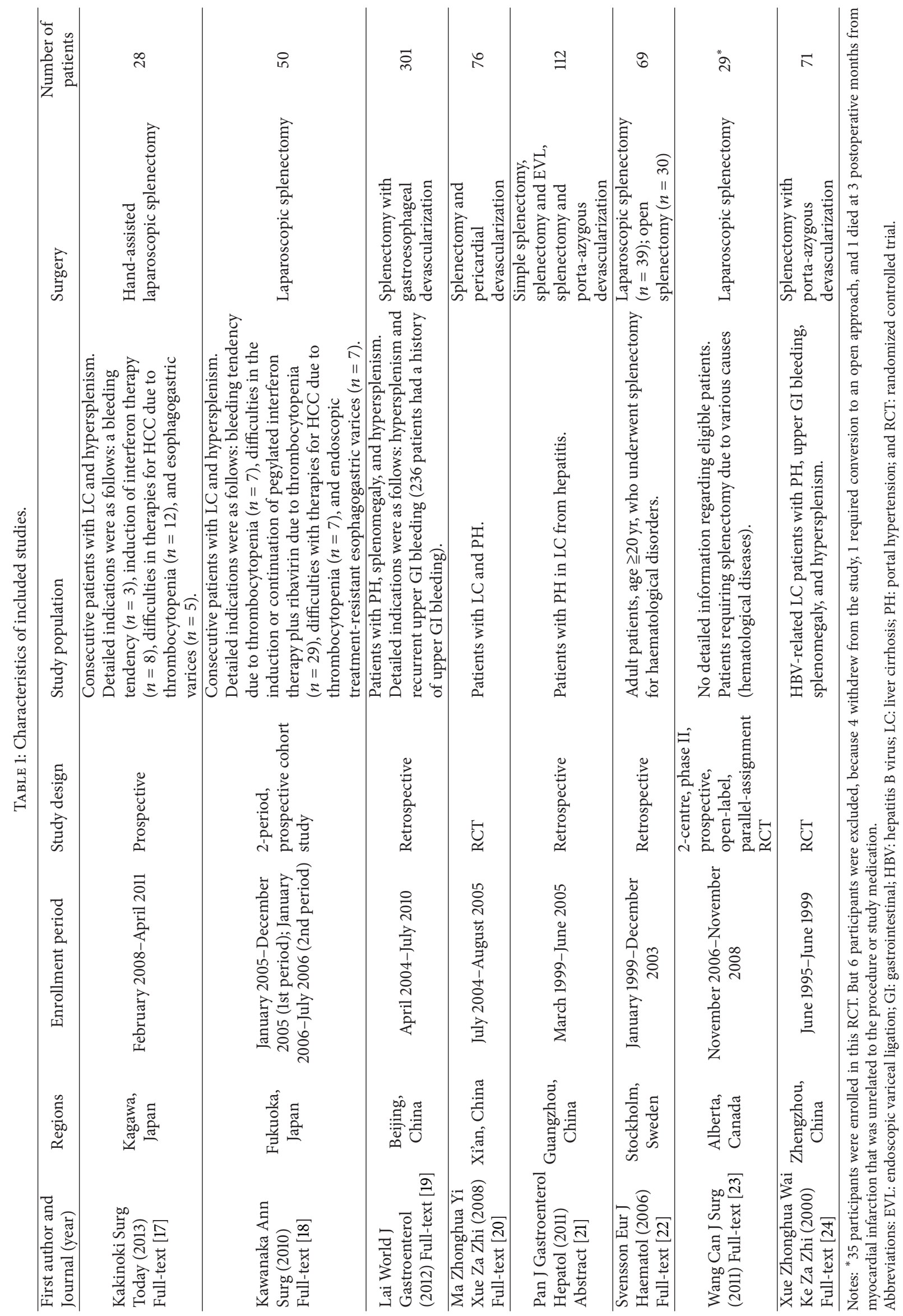




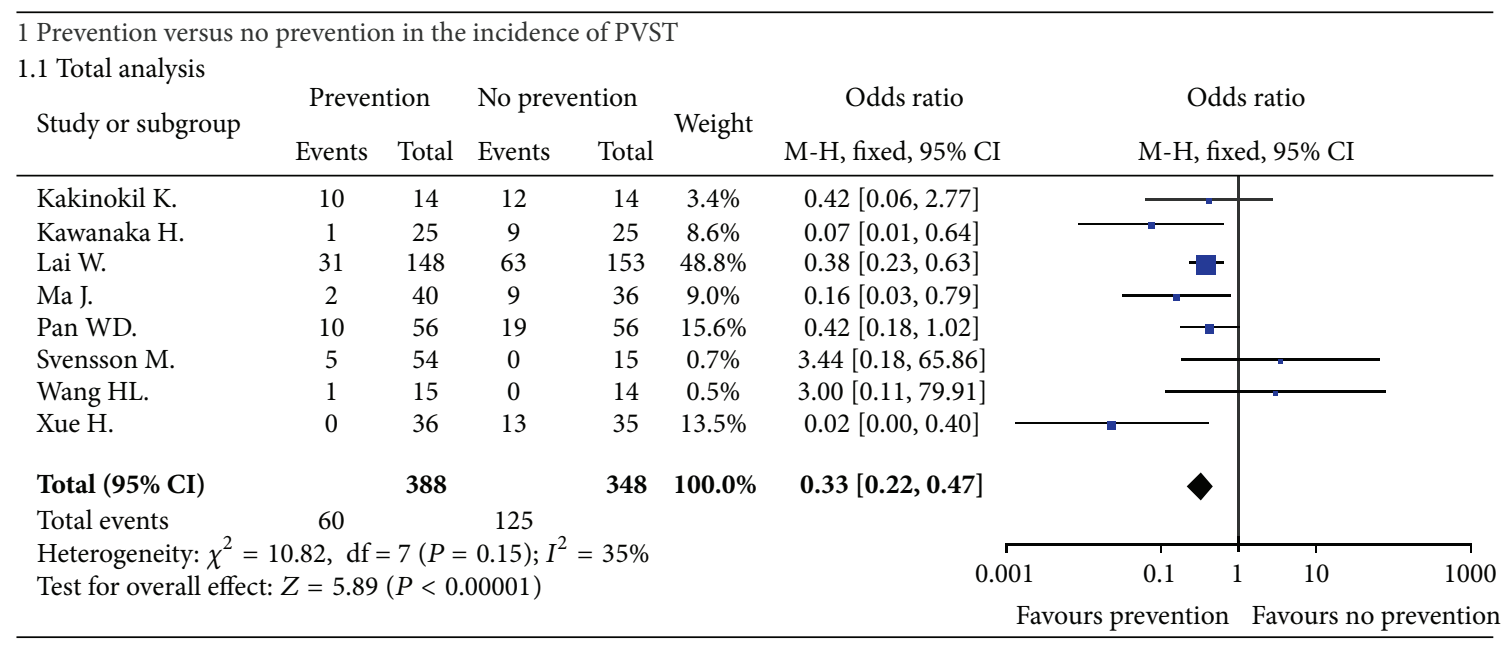

FIGURE 2: Forest plots showing the results of meta-analysis comparing the incidence of portal venous system thrombosis after splenectomy between patients who received the preventive measures and those who did not.

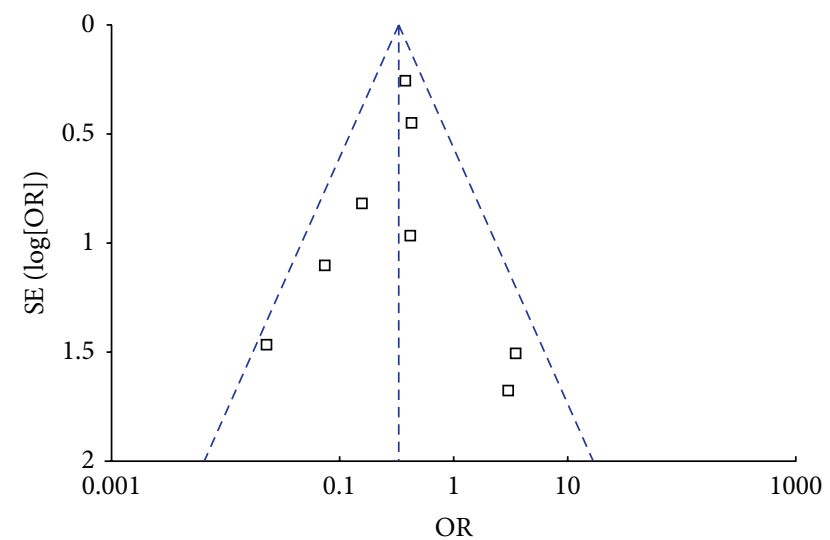

FIGURE 3: Funnel plot to explore the publication bias in the metaanalyses comparing the incidence of portal venous system thrombosis after splenectomy between patients who received the preventive measures and those who did not.

between patients who received the preventive measures and those who did not. Heterogeneity among studies was not significant $\left(I^{2}=35 \%, P=0.15\right)$. Using a fixed-effect model, the pooled OR was 0.33 (95\% CI: $0.22-0.47, P<0.00001$ ) (Figure 2), suggesting a significantly lower incidence of PVST after splenectomy in patients who received the preventive measures. Funnel plots demonstrated all studies laid within 95\%CI, suggesting no proof of publication bias (Figure 3).

\subsubsection{Subgroup Analysis according to the Type of Study} Population. In the subgroup analysis of 2 studies including the patients with hematological diseases, only anticoagulant drugs were used for the prevention of PVST. The heterogeneity among studies was not significant $\left(I^{2}=0 \%, P=0.95\right)$. Using a fixed-effect model, the pooled OR was 3.27 (95\% CI: $0.36-29.57, P=0.29$ ) (Supplementary Figure 1), suggesting that the use of anticoagulants might not significantly decrease the incidence of PVST after splenectomy in patients with hematological diseases.

In the subgroup analysis of 6 studies including the patients with portal hypertension, 3 different types of drugs were used for the prevention of PVST. The heterogeneity among studies was not significant $\left(I^{2}=28 \%, P=0.22\right)$. Using a fixed-effect model, the pooled OR was 0.29 (95\% CI: $0.20-$ $0.43, P<0.00001$ ) (Supplementary Figure 1), suggesting that the implementation of pharmacologic prophylaxis could significantly reduce the incidence of PVST after splenectomy in patients with portal hypertension.

3.2.2. Subgroup Analysis according to the Type of Drugs Used for the Prevention of PVST. In the subgroup analysis of 6 studies using anticoagulants, the heterogeneity was not significant $\left(I^{2}=15 \%, P=0.32\right)$. Using a fixed-effect model, the pooled OR was 0.40 (95\% CI: $0.27-0.59, P<0.00001$ ) (Supplementary Figure 2), suggesting that the use of anticoagulants could significantly decrease the incidence of PVST after splenectomy.

In the subgroup analysis of 1 study using thrombolytics, the pooled OR was 0.02 (95\% CI: $0.001-0.40, P=0.010$ ) (Supplementary Figure 2), suggesting that the use of thrombolytics could significantly decrease the incidence of PVST after splenectomy.

In the subgroup analysis of 1 study using prostaglandin E1, the pooled OR was 0.16 (95\% CI: $0.03-0.79, P=$ 0.02) (Supplementary Figure 2), suggesting that the use of prostaglandin El could significantly decrease the incidence of PVST after splenectomy.

3.2.3. Subgroup Analysis according to the Study Design. In the subgroup analysis of 3 randomized studies, the heterogeneity was significant $\left(I^{2}=59 \%, P=0.09\right)$. Using a random-effect model, the pooled OR was 0.19 (95\% CI: $0.02-1.89, P=0.16$ ) (Supplementary Figure 3), suggesting a similar incidence of PVST after splenectomy between the two groups. 


\begin{tabular}{|c|c|c|c|c|c|c|c|c|c|}
\hline \multicolumn{10}{|c|}{2 Prevention versus no prevention in the risk of bleeding } \\
\hline \multirow{2}{*}{ Study or subgroup } & \multicolumn{2}{|c|}{ Prevention } & \multicolumn{2}{|c|}{ No prevention } & \multirow{2}{*}{ Weight } & Odds ratio & \multicolumn{3}{|c|}{ Odds ratio } \\
\hline & Events & Total & Events & Total & & M-H, fixed, 95\% CI & \multicolumn{3}{|c|}{ M-H, fixed, 95\% CI } \\
\hline Lai W. & 1 & 148 & 2 & 153 & $66.9 \%$ & $0.51[0.05,5.73]$ & & & \\
\hline Wang HL. & 1 & 15 & 1 & 14 & $33.1 \%$ & $0.93[0.05,16.42]$ & & & \\
\hline Total $(95 \% \mathrm{CI})$ & & 163 & & 167 & $100.0 \%$ & $0.65[0.10,4.04]$ & & & \\
\hline Total events & 2 & & 3 & & & & & & \\
\hline \multicolumn{7}{|c|}{ Heterogeneity: $\chi^{2}=0.10, \mathrm{df}=1(P=0.76) ; I^{2}=0 \%$} & $\begin{array}{l}0.01 \quad 0.1 \\
\text { Favours prevention }\end{array}$ & 1 Favours $n$ & $\begin{array}{l}10 \quad 100 \\
\text { no prevention }\end{array}$ \\
\hline
\end{tabular}

FIGURE 4: Forest plots showing the results of meta-analysis comparing the risk of bleeding between patients who received the preventive measures and those who did not.

In the subgroup analysis of 5 nonrandomized studies, the heterogeneity was not significant $\left(I^{2}=9 \%, P=0.35\right)$. However, given the consistency of statistical methods between the two subgroups analyses, we still used a random-effect model to calculate a more conservative result. The pooled OR was 0.38 (95\% CI: $0.24-0.63, P=0.00001$ ) (Supplementary Figure 3), suggesting a significantly lower incidence of PVST after splenectomy in patients who received the preventive measures.

3.3. Risk of Bleeding. Only 2 studies provided the data regarding the incidence of bleeding between patients who received the preventive measures and those who did not. Heterogeneity among studies was not significant $\left(I^{2}=0 \%\right.$, $P=0.67)$. Using a fixed-effect model, the pooled OR was 1.51 (95\% CI: 0.24-9.27, $P=0.66$ ) (Figure 4), suggesting a similar incidence of bleeding between the two groups. Funnel plots were not performed due to a very small number of studies included.

\section{Discussion}

Our systematic review and meta-analysis primarily aimed to compare the incidence of PVST and risk of bleeding between patients who received the preventive measures for the development of PVST after splenectomy and those who did not. A major finding of our study was that the drugs, including anticoagulants, thrombolytics, and prostaglandin E1, could significantly decrease the incidence of PVST after splenectomy. However, according to the results of subgroup analyses, we found that the efficacy of pharmacologic prophylaxis of PVST after splenectomy remained significant in patients with portal hypertension and hypersplenism but might disappear in those with hematological diseases. This unexpected finding potentially questioned the necessity of pharmacologic prophylaxis of PVST after splenectomy in patients with hematological diseases. But it should be noted that only 2 studies with a small sample size explored this issue in patients with hematological diseases. Additionally, the number of PVST events was very small in the 2 studies, which potentially hampered from achieving a statistical significance. As mentioned by Wang et al. [23], a low incidence of PVST in their study might be contributed by a relatively low proportion of participants with myeloproliferative disease, lymphoproliferative disorders, and hereditary hemolytic anemia. In addition, our meta-analysis showed that the efficacy of preventive measures was significant in nonrandomized studies, but not in randomized studies. However, as we closely looked at the results of the subgroup analysis of 3 randomized studies, there was a trend towards favoring the use of pharmacologic prophylaxis of PVST after splenectomy. In detail, 2 of 3 randomized studies have shown a significant benefit of the preventive measures after splenectomy.

Theoretically, the patients with portal hypertension and hypersplenism, especially those with a lower platelets count and undergoing abdominal surgery, have a tendency of bleeding. Under this circumstance, the use of anticoagulation or thrombolysis after splenectomy in such patients often represents a clinical challenge. By contrast, our meta-analysis demonstrated that the risk of bleeding might not be increased after the implementation of preventive measures. Notably, this finding should be interpreted with caution due to the limited data from only 2 studies.

Several important risk factors of predicting the development of PVST after splenectomy have been increasingly recognized. They mainly include a greater spleen weight, a wider portal or splenic vein diameter, a higher D-dimer and P-selectin level, use of laparoscopic technique, and presence of hemolytic anemia or myeloproliferative disorders [3-8]. Accordingly, the early implementation of pharmacologic prophylaxis of PVST after splenectomy may be more reasonable and cost-effective in patients with the abovementioned risk factors. However, in our systematic review and meta-analysis, no relevant data regarding the efficacy of preventive measures in these "high-risk" patients could be extracted from these included studies. Thus, further studies should focus on such patients.

Compared with surgical splenectomy, partial splenic artery embolization is less invasive without general anesthesia [25]. It has been gradually considered as an alternative to surgical splenectomy in patients with portal hypertension and hypersplenism $[25,26]$. Recent studies have shown that the efficacy of partial splenic artery embolization is comparable to that of splenectomy for the treatment of hypersplenism secondary to chronic liver disease [27]. Notably, the presence of PVST after partial splenic artery embolization remains 
a major postoperative complication. Whether or not the benefit of preventive measures for the development of PVST in patients undergoing splenectomy could be extrapolated to those undergoing partial splenic embolization should be further confirmed.

The major limitation of our study was the small number of studies included in our meta-analysis, which greatly limited the reliability of the findings from the meta-analyses regarding the risk of bleeding after the implementation of pharmacologic prophylaxis of PVST and the efficacy of preventive measures after splenectomy in patients with hematological diseases. Indeed, as known, the inclusion of nonrandomized studies would result in the selection and reporting bias. However, this behavior was primarily attributed to such a small number of included studies. In addition, the quality of these included studies was relatively low. Of the 3 randomized studies, 2 did not describe any detailed methods of randomization and sample size calculation and 1 was aborted in which the sample size needed for an adequate power was not met due to the low enrollment. Given these study limitations, the findings must be interpreted with caution.

\section{Conclusion}

The implementation of pharmacologic prophylaxis could significantly decrease the incidence of PVST after splenectomy in patients with portal hypertension and hypersplenism and might not increase the risk of bleeding. However, owing to the limited evidence from 2 studies, the efficacy of anticoagulation for the prevention of PVST after splenectomy in patients with hematological diseases remained questioned. Thus, further well-designed randomized studies in different study population should be warranted to provide a confirmative recommendation.

\section{Conflict of Interests}

The authors declare that there is no conflict of interests regarding the publication of this paper.

\section{Authors' Contribution}

Xingshun Qi conceived and drafted the paper. Xingshun Qi and Ming Bai performed the literature search and selection, data extraction, quality assessment, and statistical analyses; Xiaozhong Guo and Daiming Fan gave critical comments and revised the paper. All authors have made an intellectual contribution to the paper and approved the submission.

\section{References}

[1] V. De Stefano and I. Martinelli, "Splanchnic vein thrombosis: clinical presentation, risk factors and treatment," Internal and Emergency Medicine, vol. 5, no. 6, pp. 487-494, 2010.

[2] D.-C. Valla and B. Condat, "Portal vein thrombosis in adults: pathophysiology, pathogenesis and management," Journal of Hepatology, vol. 32, no. 5, pp. 865-871, 2000.

[3] M. Ikeda, M. Sekimoto, S. Takiguchi et al., "High incidence of thrombosis of the portal venous system after laparoscopic splenectomy: a prospective study with contrast-enhanced CT scan," Annals of Surgery, vol. 241, no. 2, pp. 208-216, 2005.

[4] E. R. Winslow, L. M. Brunt, J. A. Drebin, N. J. Soper, M. E. Klingensmith, and E. Dunn, "Portal vein thrombosis after splenectomy," The American Journal of Surgery, vol. 184, no. 6, pp. 631-635, 2002.

[5] K. M. Stamou, K. G. Toutouzas, P. B. Kekis et al., "Prospective study of the incidence and risk factors of postsplenectomy thrombosis of the portal, mesenteric, and splenic veins," Archives of Surgery, vol. 141, no. 7, pp. 663-669, 2006.

[6] L. Wang, G.-J. Liu, Y.-X. Chen, H.-P. Dong, Y.-Q. Zhang, and L.-X. Wang, "Combined use of D-dimer and P-selectin for the diagnosis of splenic or portal vein thrombosis following splenectomy," Thrombosis Research, vol. 125, no. 5, pp. e206e209, 2010.

[7] K. Danno, M. Ikeda, M. Sekimoto et al., "Diameter of splenic vein is a risk factor for portal or splenic vein thrombosis after laparoscopic splenectomy," Surgery, vol. 145, no. 5, pp. 457-466, 2009.

[8] F. Romano, R. Caprotti, M. Conti et al., "Thrombosis of the splenoportal axis after splenectomy," Langenbeck's Archives of Surgery, vol. 391, no. 5, pp. 483-488, 2006.

[9] A. Morabia, "Heparin doses and major bleedings," The Lancet, vol. 1, no. 8492, pp. 1278-1279, 1986.

[10] M. Zidane, M. T. Schram, E. W. Planken et al., "Frequency of major hemorrhage in patients treated with unfractionated intravenous heparin for deep venous thrombosis or pulmonary embolism: a study in routine clinical practice," Archives of Internal Medicine, vol. 160, no. 15, pp. 2369-2373, 2000.

[11] N. J. G. M. Veeger, M. Piersma-Wichers, J. G. P. Tijssen, H. L. Hillege, and J. van der Meer, "Individual time within target range in patients treated with vitamin $\mathrm{K}$ antagonists: Main determinant of quality of anticoagulation and predictor of clinical outcome. A retrospective study of 2300 consecutive patients with venous thromboembolism," British Journal of Haematology, vol. 128, no. 4, pp. 513-519, 2005.

[12] D. Moher, A. Liberati, J. Tetzlaff, and D. G. Altman, "ReprintPreferred Reporting items for systematic reviews and metaanalyses: the PRISMA statement," Physical Therapy, vol. 89, no. 9, pp. 873-880, 2009.

[13] G. A. Wells, B. Shea, D. O'Connell et al., “The NewcastleOttawa Scale (NOS) for assessing the quality of nonrandomised studies in meta-analyses," http://www.ohri.ca/programs/ clinical_epidemiology/oxford.asp.

[14] N. Mantel and W. Haenszel, "Statistical aspects of the analysis of data from retrospective studies of disease," Journal of the National Cancer Institute, vol. 22, no. 4, pp. 719-748, 1959.

[15] R. DerSimonian and N. Laird, "Meta-analysis in clinical trials," Controlled Clinical Trials, vol. 7, no. 3, pp. 177-188, 1986.

[16] J. P. T. Higgins, S. G. Thompson, J. J. Deeks, and D. G. Altman, "Measuring inconsistency in meta-analyses," The British Medical Journal, vol. 327, no. 7414, pp. 557-560, 2003.

[17] K. Kakinoki, K. Okano, H. Suto et al., "Hand-assisted laparoscopic splenectomy for thrombocytopenia in patients with cirrhosis," Surgery Today, vol. 43, no. 8, pp. 883-888, 2013.

[18] H. Kawanaka, T. Akahoshi, N. Kinjo et al., "Impact of antithrombin III concentrates on portal vein thrombosis after splenectomy in patients with liver cirrhosis and hypersplenism," Annals of Surgery, vol. 251, no. 1, pp. 76-83, 2010.

[19] W. Lai, S.-C. Lu, G.-Y. Li et al., "Anticoagulation therapy prevents portal-splenic vein thrombosis after splenectomy with 
gastroesophageal devascularization," World Journal of Gastroenterology, vol. 18, no. 26, pp. 3443-3450, 2012.

[20] J.-C. Ma, J. Zhao, Q.-H. Su, D.-H. Zhang, Y. Guo, and Z.-Z. Ji, "Effects of alprostadil in prevention of portal vein thrombogenesis after splenectomy and devascularization: a clinical observation of 76 patients with portal hypertension," National Medical Journal of China, vol. 88, no. 8, pp. 524-526, 2008.

[21] W. D. Pan and R. Y. Xu, "Precaution of the portal system thrombosis in patients with portal hypertension of cirrhosis resulting from hepatitis after splenectomy," Journal of Gastroenterology and Hepatology, vol. 26, article 140, 2011.

[22] M. Svensson, M. Wirén, E. Kimby, and H. Hägglund, "Portal vein thrombosis is a common complication following splenectomy in patients with malignant haematological diseases," European Journal of Haematology, vol. 77, no. 3, pp. 203-209, 2006.

[23] H. Wang, D. Kopac, R. Brisebois, C. Sample, and A. M. J. Shapiro, "Randomized controlled trial to investigate the impact of anticoagulation on the incidence of splenic or portal vein thrombosis after laparoscopic splenectomy," Canadian Journal of Surgery, vol. 54, no. 4, pp. 227-231, 2011.

[24] H. Xue, H. Zhang, Y. Zhang, and Q. Jiang, "Portal anticoagulation in preventing thrombosis after porta-azygous devascularization for portal hypertension," Zhonghua Wai Ke Za Zhi, vol. 38, no. 11, pp. 855-857, 2000.

[25] K. G. Koconis, H. Singh, and G. Soares, "Partial splenic embolization in the treatment of patients with portal hypertension: a review of the english language literature," Journal of Vascular and Interventional Radiology, vol. 18, no. 4, pp. 463481, 2007.

[26] B. Sangro, I. Bilbao, I. Herrero et al., "Partial splenic embolization for the treatment of hypersplenism in cirrhosis," Hepatology, vol. 18, no. 2, pp. 309-314, 1993.

[27] M. A. Amin, M. M. El Gendy, I. E. Dawoud, A. Shoma, A. M. Negm, and T. A. Amer, "Partial splenic embolization versus splenectomy for the management of hypersplenism in cirrhotic patients," World Journal of Surgery, vol. 33, no. 8, pp. 1702-1710, 2009. 


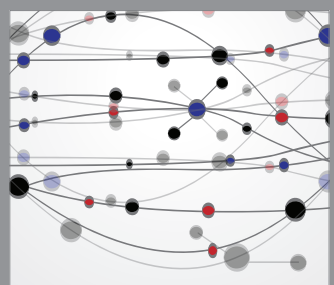

The Scientific World Journal
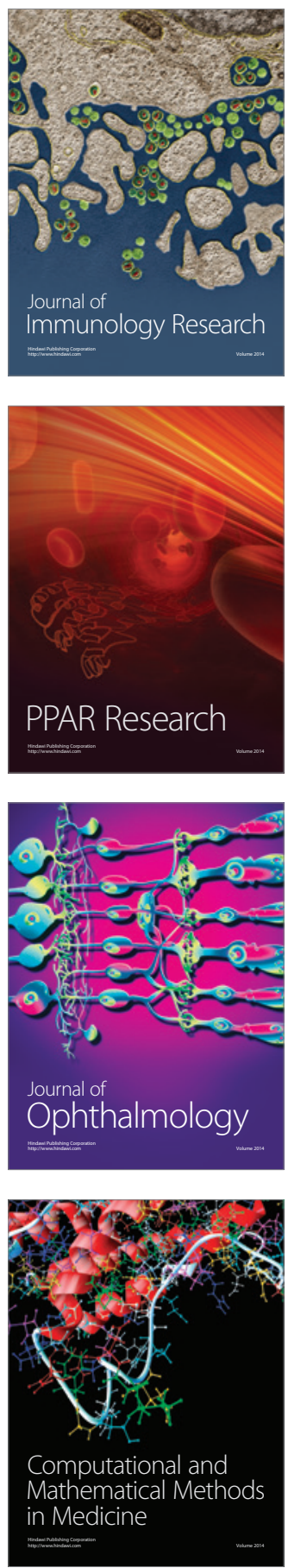

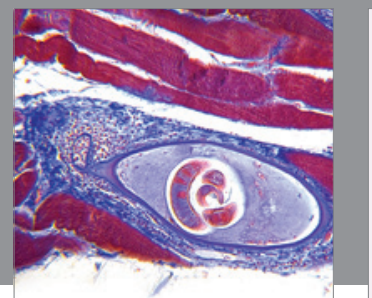

Gastroenterology

Research and Practice
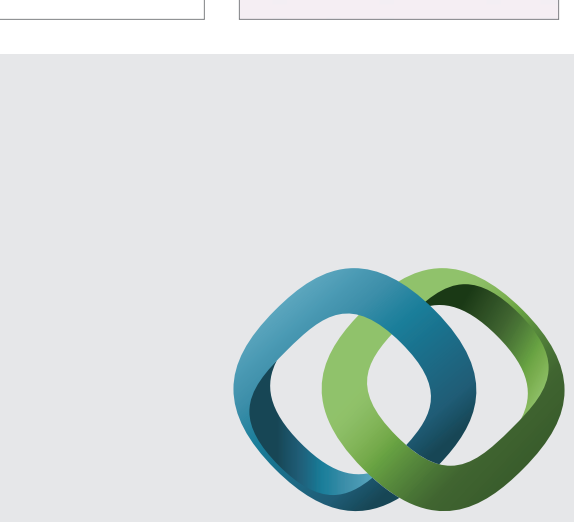

\section{Hindawi}

Submit your manuscripts at

http://www.hindawi.com
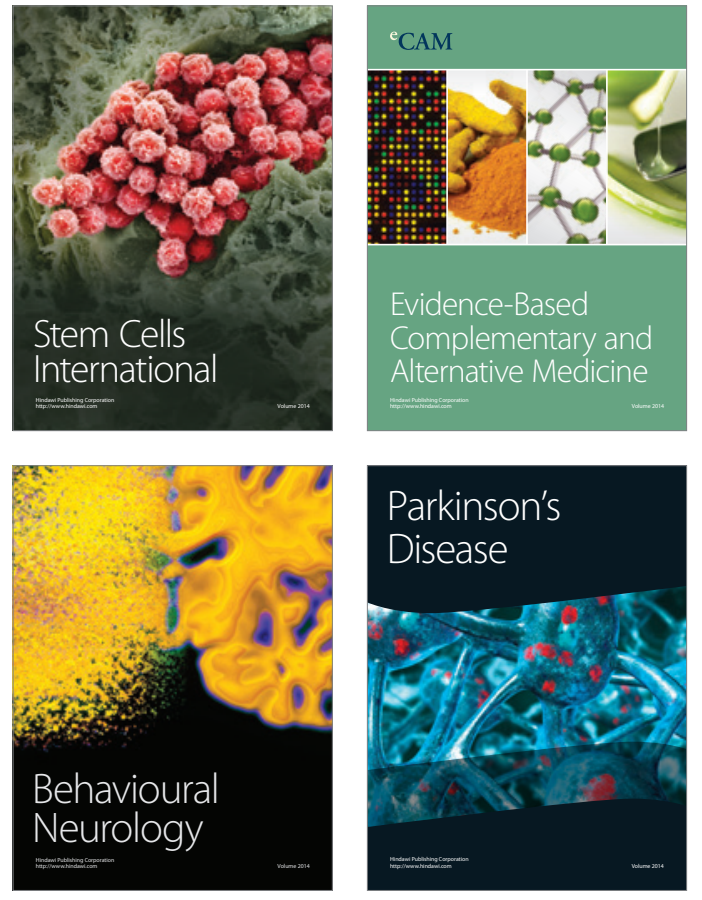
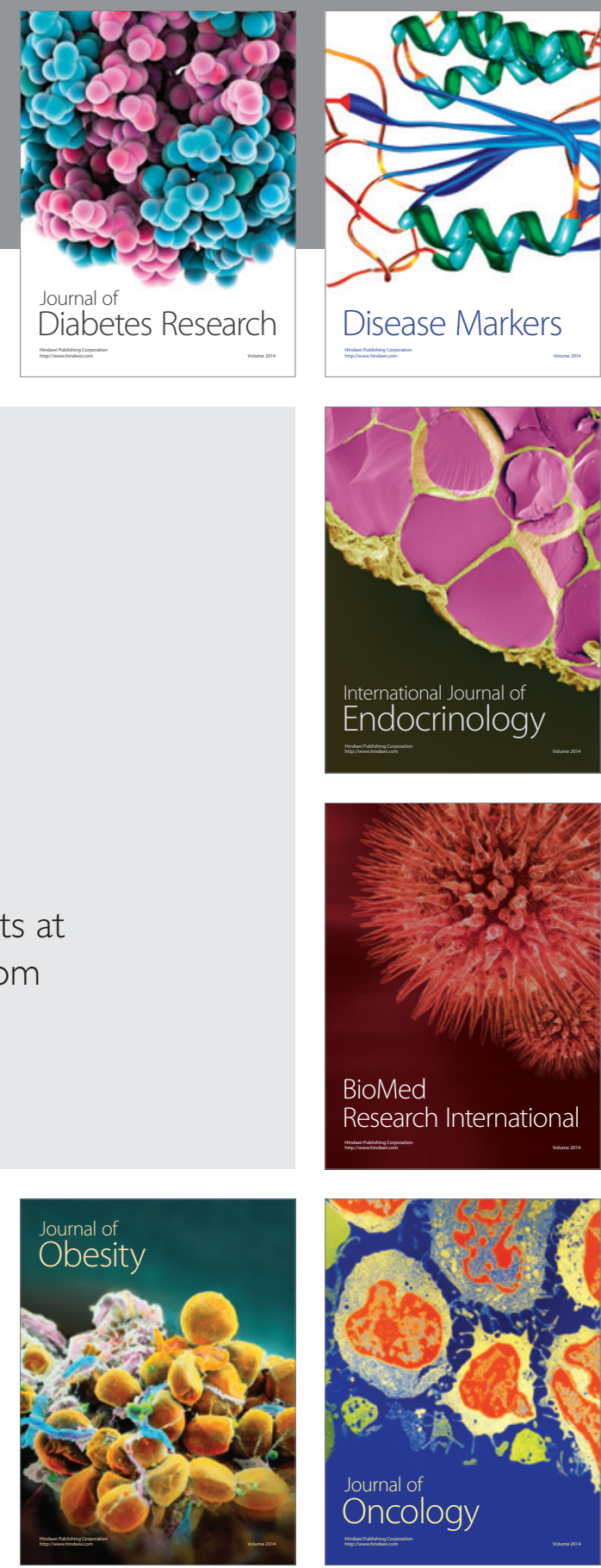

Disease Markers
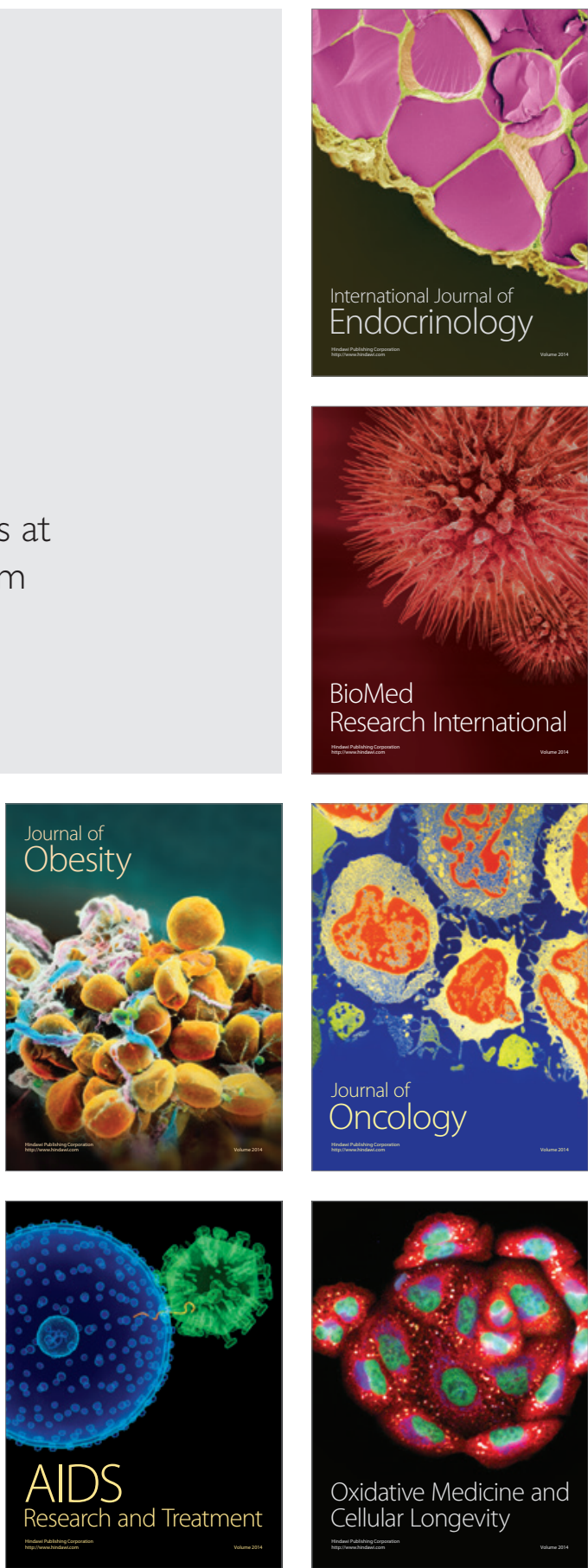